\title{
A reconfigurable architecture for object detection using adaptive threshold
}

\section{Sangeeta. M. Gangannavar ${ }^{1 *}$, S. S. Navalgund ${ }^{2}$ and Satish S. Bhairannawar ${ }^{3}$}

M. Tech Student, Department of Electronics and Communication Engineering, SDM College of Engineering and Technology, Dharwad, Karnataka, India ${ }^{1}$

Assistant Professor, Department of Electronics and Communication Engineering, SDM College of Engineering and Technology, Dharwad, Karnataka, India ${ }^{2}$

Professor, Department of Electronics and Communication Engineering, SDM College of Engineering and Technology, Dharwad, Karnataka, India ${ }^{3}$

Received: 20-June-2018; Revised: 30-August-2018; Accepted: 05-September-2018

(C)2018 ACCENTS

\begin{abstract}
The detection of objects is important in many computer vision applications. This paper proposes a reconfigurable architecture for object detection using adaptive threshold with an efficient algorithm for removal of salt and pepper noise from the colour and grayscale images. The main objective of this paper is to design an alternate architecture of object detection using adaptive threshold. In this paper, a type median filter is used to preserve the edges and to reduce the salt and pepper noise easily of the input and reference image is discussed. The pre-processed images are applied to $2 D$ discrete wavelet transform (2D-DWT) to remove variable illumination and to select appropriate sub-band, i.e., low-low (LL) band which contains maximum information of the original image. The modified background subtraction is used to remove the background from $L L$ band of input and reference images to obtain a foreground image. The detected object is fed to median filter to remove any small amounts of noise which is still present in the image. The modified decision based partially trimmed global median (MDBPTGM) filter was used to give better results in terms of mean square error (MSE), peak signal to noise ratio (PSNR) and image enhancement factor (IEF). Hardware parameters such as slice registers and flip-flop pairs, latches, lookup table (LUT), shift registers and memory usage were compared with the existing techniques. Propose architecture used less number of hardware parameters. It means the proposed design reduces power and the area usage in comparison to the other techniques.
\end{abstract}

\section{Keywords}

Object detection, Discrete wavelet transform, Adaptive threshold, Modified decision based partially trimmed global median filter.

\section{Introduction}

Now days, detection of objects is the first step in various applications such as video surveillance, image retrieval, vehicle navigation, and auto robot navigation, etc. Detection of objects provides the detailed information about the objects, focus on recognition of objects and describes the category or classification of the object belongs to and activity or analysis of the object. Object detection has a great variety of applications, where each application needs different algorithms, specifications, requirements and constraints. Object recognition and object detection contribute efficient tasks in computer vision applications [1]. Object detection is classified into soft detection and hard detection.

*Author for correspondence

257
Soft detection gives only information about the presence of an object, whereas hard detection provides efficient information like location, pattern, and activity of object with accuracy. In general, there are three main sections in object or face detection, namely pre-processing, feature extraction and matching section.

It is observed that the many online games, web $\mathrm{PDF}^{\mathrm{ce}} \mathrm{s}$, web pages consist of noise. These corrupted images and graphics are filtered by pre-processing technique called image filtering. This is a technique which is used as a pre-processing step in many applications such as face identification and recognition, image compression and pattern recognition [2].

The pre-processed images are compressed by discrete wavelet transform (DWT) and it is widely used in 
signals and image processing tasks. DWT is one of wavelet transforms commonly used in the image compression technique. Now a day, storage of image is commonly faced problem with many digital images based applications like security based applications, retail stores, health and hotel management systems, etc. Here, the image compression technique is used to solve these problems. The aim at image compression technique is not only focusing on reducing the amount of data required to represent the image, but also to maintain quality of image by preserving the information about the images [3]. DWT has a wide range of applications for biological field like cell membrane recognition, health applications such as deoxyribonucleic acids (DNA) analysis, heart rate and electrocardiogram (ECG) analysis, DNA analysis, protein analysis, security based applications like speech recognition and fingerprint verification [4]. Image processing operates on pixel level. It is divided into three categories to obtain a modified version of the image. These are reconstruction, transformation and classification. Classification is further divided into two sub-types, segmentation which means the process of partitioning the image into sub-parts, depends on its application. Another type of classification is defined on pixel level of gray value [5].

This paper proposes a reconfigurable architecture for object detection using adaptive threshold with an efficient algorithm for removal of salt and pepper noise from the colour and grayscale images. Salt and pepper noise are forms of noise, commonly known as impulse noise. This type of noise caused by dead pixels, analog to digital converter errors and bit errors in transmission. Image pre-processing is a vital task in image processing. In this paper, a method of image denoising based on MDBPTGM filter [6] is used to preserve the edges and to reduce the salt and pepper noise easily. The pre-processed images are applied to 2D-DWT to remove variable illumination and to select appropriate sub-band, i.e., the LL band which contains maximum information of the original image. The modified background subtraction is used to remove the background from LL band of input and reference images to obtain a foreground image. The detected object is fed to median filter to remove any small amounts of noise still present in the image. MDBPTGM filter [6] gives better results in terms of MSE, PSNR and IEF.

Sarkar et al. [7] proposed an adaptive threshold based field-programmable gate array (FPGA) implementation for object and face detection, detect moving object and face effectively by using the preprocessing block, modified background subtraction and adaptive threshold techniques. The face detection can also be performed with the same architecture by additional matching and global threshold unit, the performance parameters are better in the architecture compared to existing architectures in terms of software and hardware results. Modified decision based partially trimmed global median filter algorithm [6] explained by considering central pixel as processing pixel in $3 \times 3$ window. This algorithm compared with different types of median filters. A survey conducted on non-linear filtering techniques to remove the noise [7]. This survey covers different types of median filter with their drawbacks. Joy and Prasad [8] conducted survey on background modeling and foreground detection methods. This paper contributes to the various methodologies for foreground and background subtraction methods. In 2015, Zaitoun and Aqel [9] compares the basic block based image segmentation techniques and conclude that there is no universal segmentation technique for all kinds of images.

This paper covers the region based and edge or boundary based image segmentation. It is based on a heterogeneous system-on-chip (SoC) platform, real time object method was proposed by Qiu et al.[10], This paper synthesis on Altera Quartus II 13.1 electronic design automation (EDA) tool and it uses 3190736 block memory bits 9301 registers and 333 pins. In [11] based on ZYNQ flexible platform, object recognition and tracking method were proposed. This system uses 32984 flip-flops, 29792 LUT's, 870 memory LUT's, 77 BRAM's, 156 DSP 48 and 6 BUFG's. In [12] various image processing algorithms are used to track the moving object. This is implemented on Altera Cyclone II FPGA device and uses 6212 registers, 426 pins, 211872 memory bits and 1 PLL. In [13] a system proposed to detect the animals, humans and vehicles. This system even supports the object detection in under water and uses the 14685 slice registers, 28818 flip-flops, 27 BRAM's, 28347 LUT's, 56 IOB's and 15 DSP48.

Section 2 describes materials and methods of the proposed architecture. Section 3 covers the results analysis of the proposed architecture and presents the simulation results. Section 4 includes comparative discussion. Section 5 deals with the conclusion.

\section{Materials and methods}

The architecture of the object detection is shown in Figure 1, where the input images are "background 
image" and object to the same background as "object image" are pre-processed using the median filter to remove the salt and pepper noise from images. The type of median filter here used is a MDBPTGM filter
[6]. The algorithm used to remove the salt and pepper noise as is discussed below and it is processed as per the block given in Figure 2.

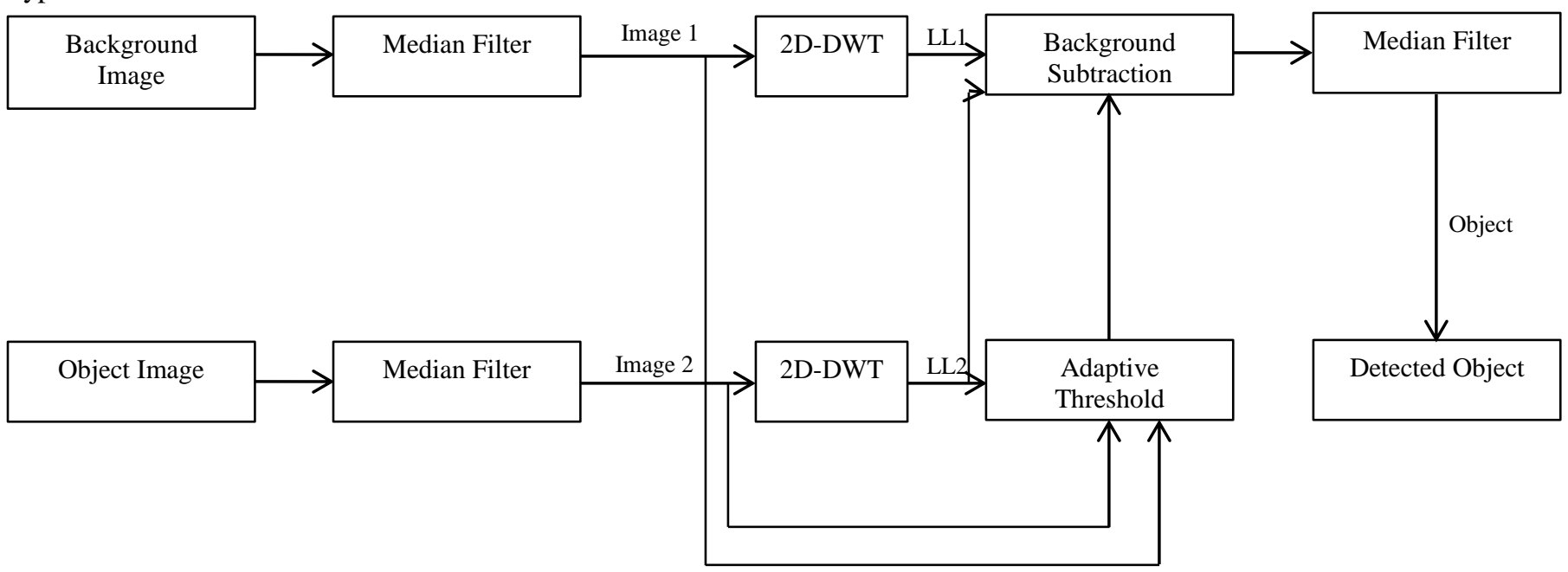

Figure 1 Proposed architecture for object detection

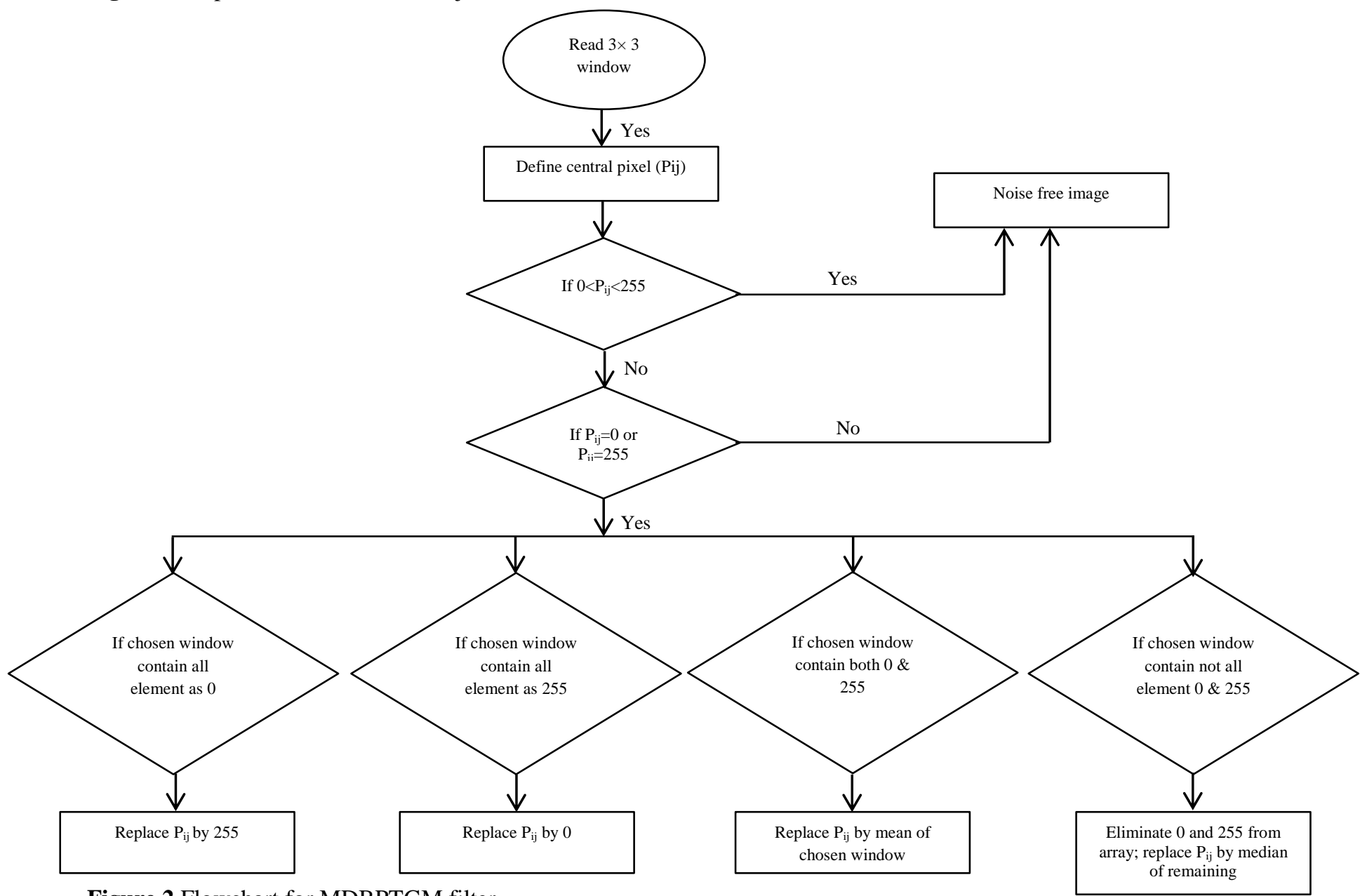

Figure 2 Flowchart for MDBPTGM filter 
There are many different types of median filters [14] to remove salt and pepper noise from the image. MDBPTGM filter [6] is a quick and efficient algorithm. In this algorithm initial $3 \times 3$ window is considered as processing window and centre pixel of the processing window treated as processing pixel $\left(\mathrm{P}_{\mathrm{ij}}\right)$. Based on this centre pixel value only it is decided that whether image is noisy or noise free. If chosen processing window contain centre pixel $\left(\mathrm{P}_{\mathrm{ij}}\right)$ value in between the salt noise (255) and pepper noise (0), then centre pixel will be considered as uncorrupted pixel and image will be declared as noise free image. It will remain unchanged. If centre pixel value is equal to 255 or 0 , then following cases are possible.

Case 1: If selected $3 \times 3$ processing window has all pixel value as zeros, then $\mathrm{P}_{\mathrm{ij}}$ replace by 255 i.e. salt noises.

Case 2: If processing window has all pixel value as 255 , then $\mathrm{P}_{\mathrm{ij}}$ replaced by 0 i.e. pepper noise.

Case 3: If the selected $3 \times 3$ processing window has both 0 's and 255's, mean of the window is calculated and replace $\mathrm{P}_{\mathrm{ij}}$ by calculated mean value.

Case 4: If the above all cases fail, it means that selected processing window has not all the element 0 and 255, then eliminate 0 's and 255's from window and find the median value of the remaining elements in the array, replace $\mathrm{P}_{\mathrm{ij}}$ with median value of selected window.

Repeat the above procedure until the whole image will be noise free. The block of MDBPTGM filter [6] is as shown in Figure 2. The filtered images are applied to DWT; it is one of image compression technique.

The pre-processed images are image 1, image 2 are compressed by using DWT. LL sub-band is obtained for both pre-processed images, which have approximate information about input images. The generated sub-bands are LL1 and LL2 for "background image" and "object image" respectively are applied to extract the foreground image (object image) by comparing with adaptive threshold value. Adaptive threshold value calculated by pixel value difference $\left(\right.$ PIXEL $\left._{\text {diff }}\right)$ between background image and object image is given in Equation 1.

PIXEL $_{\text {diff }}=\frac{\left(\left[\mathrm{B}_{1}-\mathrm{O}_{1}\right]^{2}+\left[\mathrm{B}_{1}-\mathrm{O}_{1}\right]^{2}+\left[\mathrm{B}_{1}-\mathrm{O}_{1}\right]^{2} \ldots\left[\mathrm{B}_{1}-\mathrm{O}_{1}\right]^{2}\right)}{8 \mathrm{~N}}(1)$

Where $\mathrm{B}_{1} \mathrm{~B}_{2} \ldots \ldots \ldots \ldots . . \mathrm{B}_{\mathrm{N}}$ is pixel values of the background image, $\mathrm{O}_{1}, \mathrm{O}_{2} \ldots \ldots \ldots \ldots . . \mathrm{O}_{\mathrm{N}}$ are pixel values of object image and $\mathrm{N}$ is the total dimension of the image.

Adaptive threshold $\left(\mathrm{AT}_{\mathrm{TH}}\right)$ value is calculated using the value $(\mathrm{V})$, it is obtained by pixel difference value and an LL band coefficient of object or foreground image $\left(\mathrm{LL}_{2}\right)$ is given in Equation 2.

$\mathrm{V}=\mathrm{PIXEL}_{\text {diff }}+\mathrm{LL}_{2}$

The mean of the above Equation 2 is added with an LL band coefficient of the object or foreground image $\left(\mathrm{LL}_{2}\right)$ is given in Equation 3.

$\mathrm{AT}_{\mathrm{TH}}=\operatorname{Mean}(\mathrm{V})+\mathrm{LL}_{2}$

In order to extract the foreground image, we have to remove the background from an image. By subtracting LL band coefficients of foreground image $\left(\mathrm{LL}_{2}\right)$ from the background image $\left(\mathrm{LL}_{1}\right)$ is given in (4).

$\mathrm{SUB}=\mathrm{LL}_{1}-\mathrm{LL}_{2}$

Equation 4 is obtained by subtracting the maximum and minimum pixel value between LL band coefficients of foreground and background images. The subtracted value is compared with the adaptive threshold value $\left(\mathrm{AT}_{\mathrm{TH}}\right)$, if the pixel of SUB value is greater than or equal to adaptive threshold values, then it will be taken as foreground image, otherwise it will remain as the background image is shown in below Figure 3.

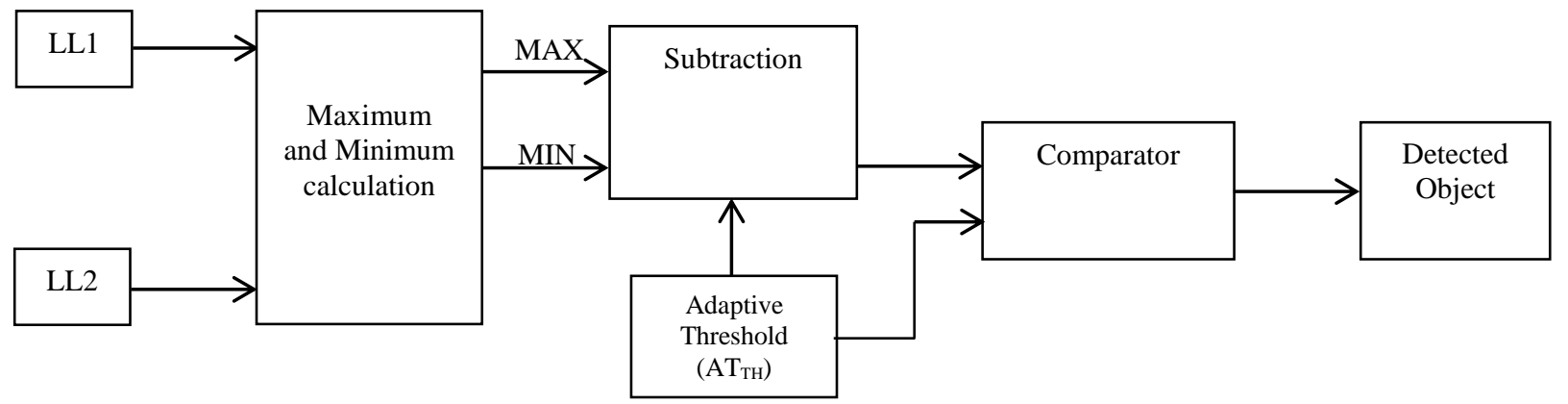

Figure 3 Block diagram for background subtraction 


\section{Results}

As discussed earlier, MDBPTGM filter was used to remove salt and pepper noise from images. This filter is simulated with the help of MATLAB R2012a and Windows 7 operating system. The performance evaluation was performed for the image "cameraman.tif" of size $256 \times 256$. It is corrupted with different noise density, varies from $10 \%$ to $100 \%$. It is necessary to check the quality images which are pre-processed by applying the MDBPTGM filter algorithm by statistical parameters like PSNR, MSE and IEF.

MSE is defined as square of difference between the original and reconstructed image. Noise density of image is directly proportional to mean square error, and results the low quality image. It is shown in Figure 4. Quality of image is improved by using MDBPTGM filter is compared with standard median filter is performed on "cameraman.tif " of size $256 \times 256$. It is shown in Figure 4 , where $\mathrm{X}$ axis represents the noise density and $\mathrm{Y}$ axis represents the MSE values. We know that MSE measures the amount errors by comparing original and reconstructed image. It is shown in Equation 5.

MSE $=\sum i j \frac{\left(Y_{i j}-Z_{i j}\right)^{2}}{M * N}$

Where $Y_{i j}$ is the original image, $Z_{i j}$ reconstructed image at pixel $(i, j)$ and $\mathrm{M} \times \mathrm{N}$ is the size of the image.

As the name itself defines, the PSNR measures the peak errors.
It is the ratio of maximum possible power of an image to power of corrupting noise that is mean square error. It is shown in Equation 6. As we know that since PSNR value is inversely proportional to MSE values, it means PSNR values are directly proportional to quality of image. Graphical representation of noise density versus PSNR values for the same image is shown in Figure 5. It clearly shows the quality of image improvement by using MDBPTGM filter.

We can observe that the PSNR values of MDBPTGM filter are higher than standard median filter as shown in Figure 5, where $\mathrm{X}$ axis represents noise density and $\mathrm{Y}$ axis represents PSNR values.

$\operatorname{PSNR}(\mathrm{dB})=10 \log _{10}\left(255^{2} / \mathrm{MSE}\right)$

Performance of the proposed algorithm has been tested by using another parameter called as IEF. Initially IEF will increase as noise density increased. When it reaches maximum peak, it starts to decrease. Graphical representation of noise density versus IEF values for the same image is shown in Figure 6. IEF is the ratio of square of difference between noisy and original image to reconstructed and original image. It is shown in Equation 7.

$I E F=\frac{\sum i j\left(N_{i j}-Y_{i j}\right)^{2}}{\sum i j\left(Z_{i j}-Y_{i j}\right)}$

Where $N_{i j}$ is noisy image, $Y_{i j}$ is the original image, $Z_{i j}$ reconstructed image at pixel $(\mathrm{i}, \mathrm{j})$.

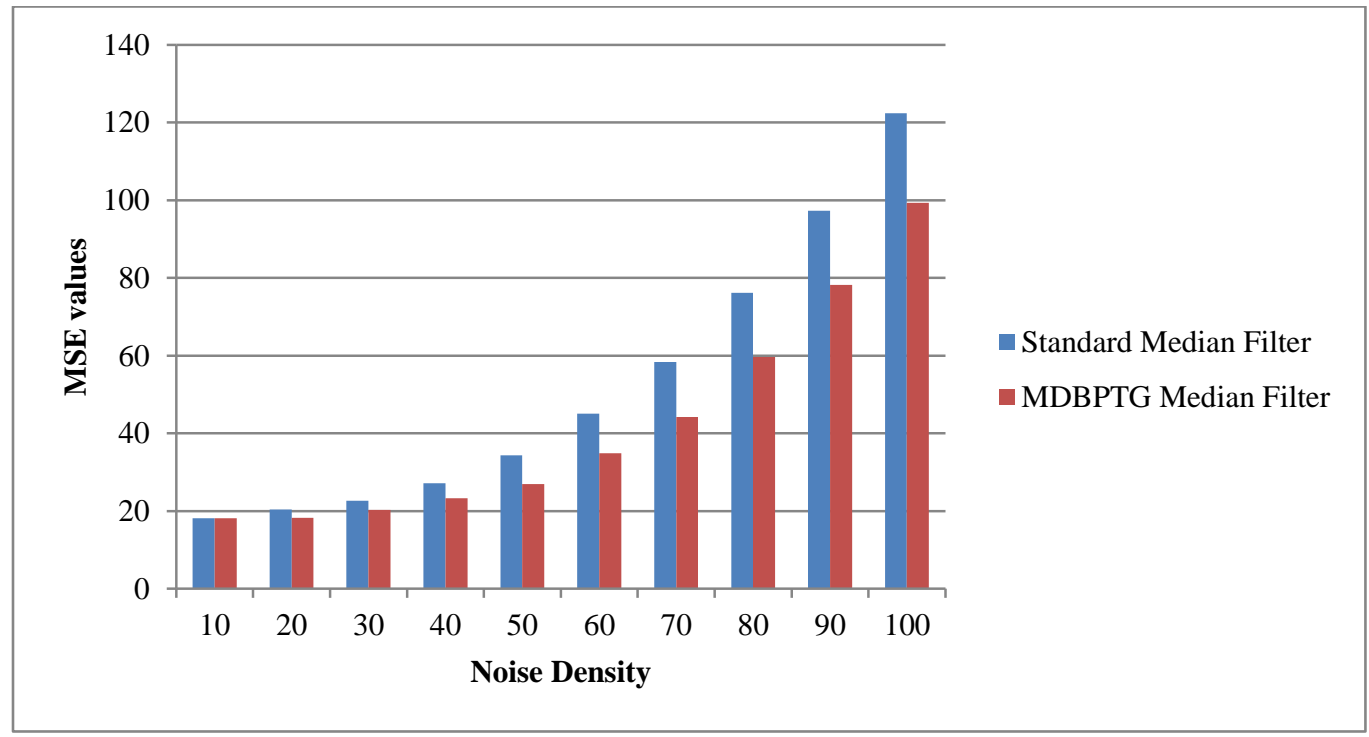

Figure 4 Noise densities versus MSE values for "cameraman.tif” image of size 256×256 


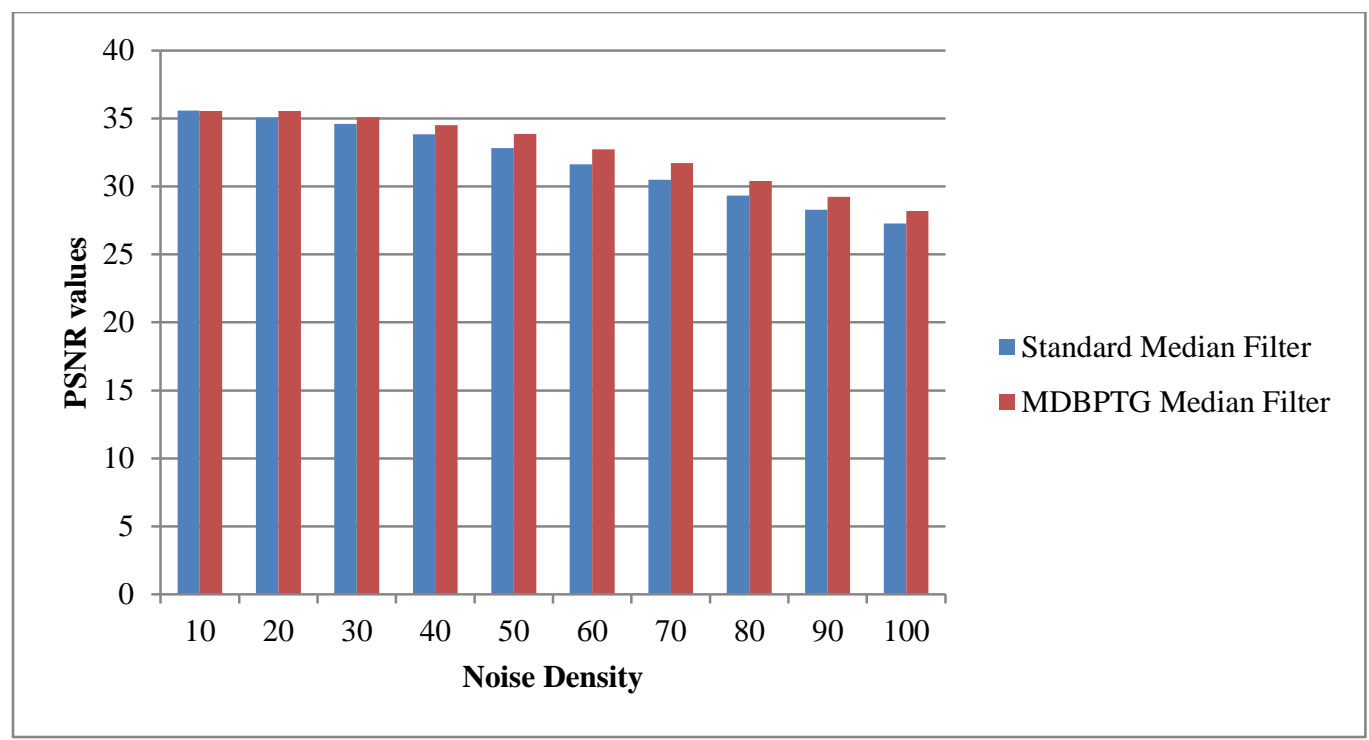

Figure 5 Noise densities versus PSNR values for "cameraman.tif" of image size $256 \times 256$

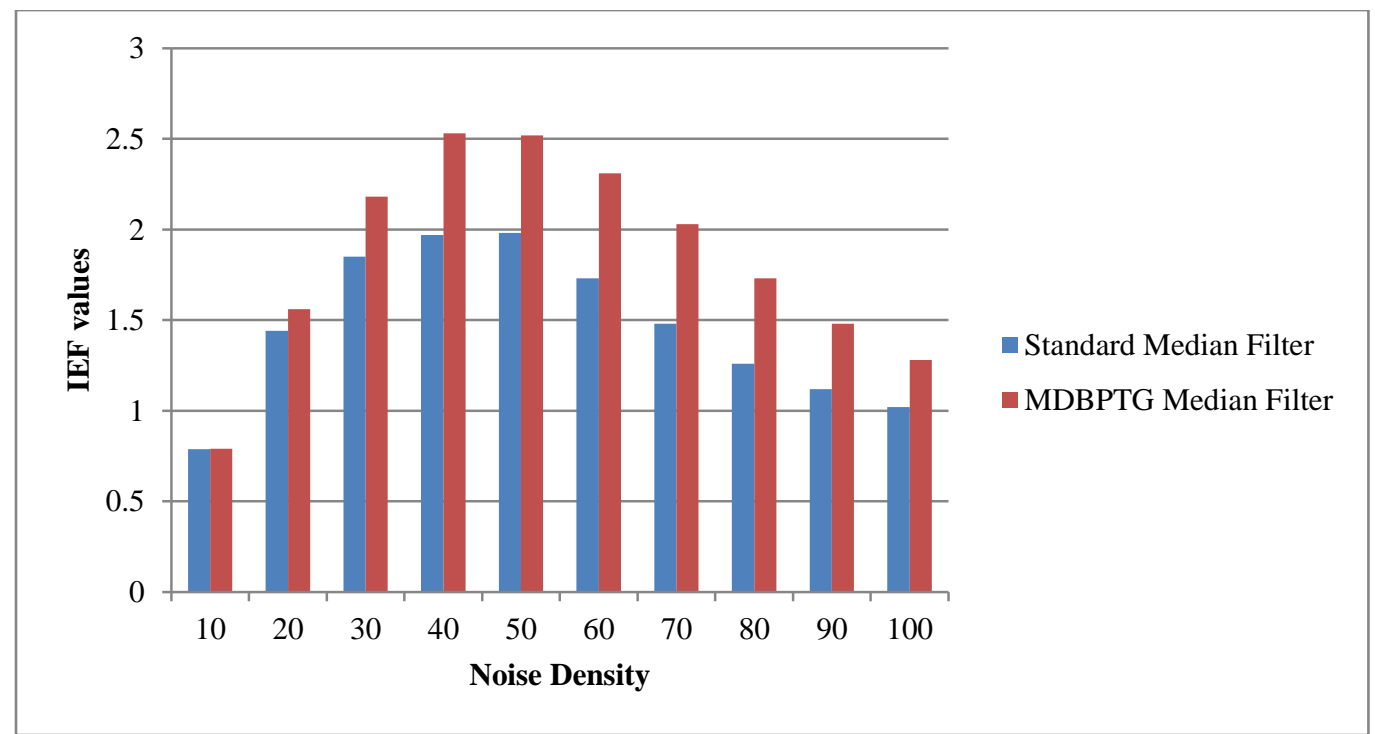

Figure 6 Noise densities versus IEF values for "cameraman.tif" image of size 256×256

The MDBPTGM filter is compared with the standard Gaussian filter with the parameter PSNR. It may be concluded that the proposed filter better to enhance the quality of the image by comparing the Gaussian filter. Graphical representation of PSNR values versus noise density is shown in Figure 7, where the $\mathrm{X}$ axis represents noise density and the $\mathrm{Y}$ axis represents PSNR values.

The experiment is conducted on the same image of "cameraman.tif" of size $256 \times 256$ and $512 \times 512$. It is corrupted by salt and pepper noise with different noise density, varies from $10 \%$ to $100 \%$. The results are shown in Table 1. Comparative results for the 262 "lena.tif" image in terms of MSE, PSNR and IEF values at different noise densities is shown in Table 2 .

It is observed that the size $512 \times 512$ shows better results in a low noise density (10\% to $50 \%)$ but image with size $256 \times 256$ gives better results at high noise density $(60 \%$ to $100 \%)$ with respect to values of MSE. Similarly with respect to the values of PSNR, image with size $512 \times 512$ shows better results in a low noise density (10\% to $50 \%$ ) but image with size $256 \times 256$ shows better results at high noise density $(60 \%$ to $100 \%)$. Graphical representation of noise density versus PSNR values is performed on 
"cameraman.tif" of size $256 \times 256$ and $512 \times 512$ are shown in Figure 8 and 9. It clearly shows that the higher dimension images provide better results at low noise density and lower dimension images provide better results in case of high noise density.

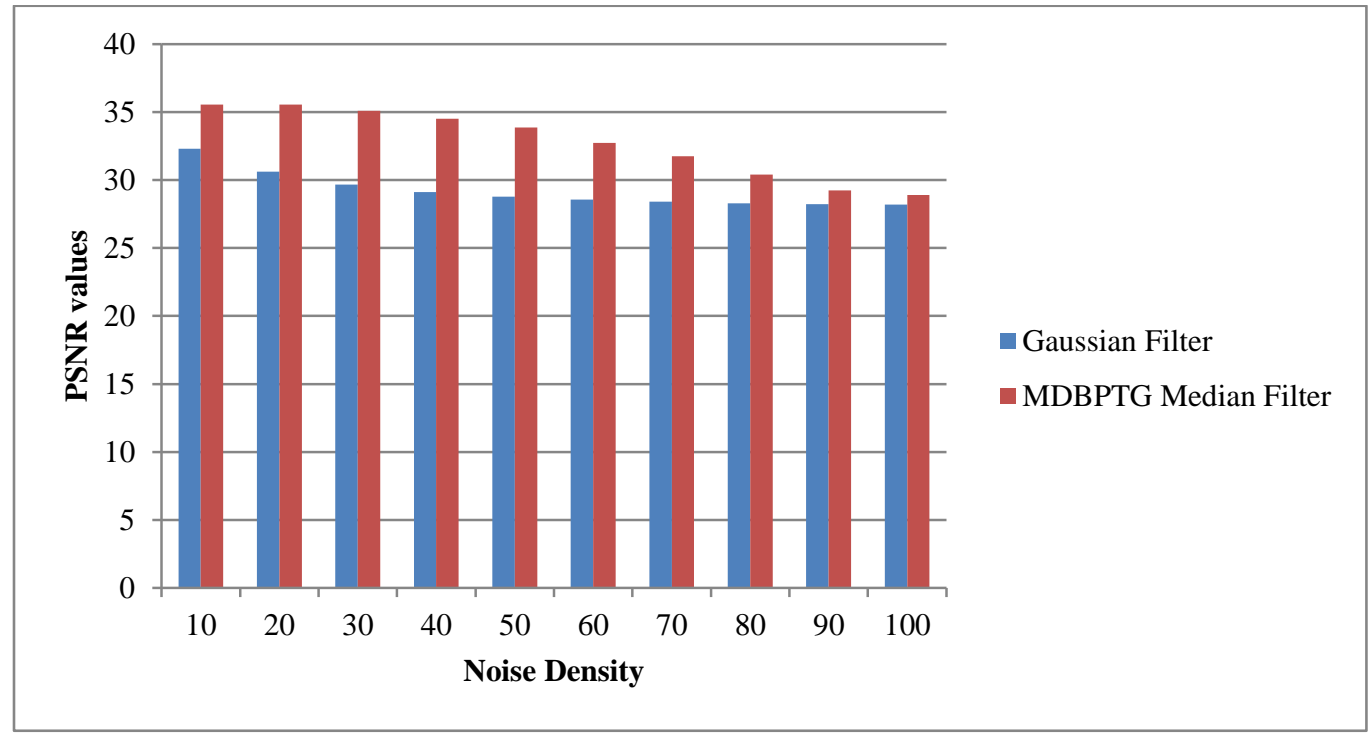

Figure 7 Results of PSNR values of Gaussian filter with MDBPTG median filter

Table 1 Comparison of MSE, PSNR and IEF values of the "cameraman.tif" image of size $256 \times 256$ and $512 \times 512$

\begin{tabular}{|c|c|c|c|c|c|c|c|}
\hline $\begin{array}{l}\text { Noise } \\
(\%)\end{array}$ & density & MSE $(512 \times 512)$ & MSE $(256 \times 256)$ & PSNR $(512 \times 512)$ & PSNR $(256 \times 256)$ & IEF $(512 \times 512)$ & IEF $(256 \times 256)$ \\
\hline 10 & & 6.37 & 18.18 & 40.11 & 35.56 & 2.47 & 0.79 \\
\hline 20 & & 8.54 & 18.20 & 38.84 & 35.56 & 3.37 & 1.56 \\
\hline 40 & & 16.79 & 23.29 & 35.91 & 34.49 & 3.27 & 2.53 \\
\hline 50 & & 24.54 & 26.92 & 34.26 & 33.86 & 2.65 & 2.54 \\
\hline 60 & & 36.07 & 34.81 & 32.59 & 32.74 & 2.08 & 2.31 \\
\hline 70 & & 51.31 & 44.15 & 31.06 & 31.71 & 1.65 & 2.03 \\
\hline 80 & & 70.88 & 59.68 & 29.65 & 30.40 & 1.35 & 1.73 \\
\hline 90 & & 93.99 & 78.16 & 28.43 & 29.23 & 1.14 & 1.48 \\
\hline 100 & & 118.18 & 99.37 & 27.43 & 28.19 & 1.00 & 1.28 \\
\hline
\end{tabular}

Table 2 Comparison of MSE, PSNR and IEF values of the "lena.tif" image of size $256 \times 256$ and $512 \times 512$

\begin{tabular}{|c|c|c|c|c|c|c|c|}
\hline $\begin{array}{l}\text { Noise } \\
(\%)\end{array}$ & Density & MSE $(512 \times 512)$ & MSE $(256 \times 256)$ & PSNR $(512 \times 512)$ & PSNR $(256 \times 256)$ & IEF $(512 \times 512)$ & IEF $(256 \times 256)$ \\
\hline 10 & & 9.83 & 17.58 & 38.23 & 35.71 & 1.54 & 0.83 \\
\hline 20 & & 12.23 & 20.69 & 37.29 & 35.00 & 2.43 & 1.39 \\
\hline 30 & & 15.54 & 24.41 & 36.27 & 34.28 & 2.71 & 1.71 \\
\hline 40 & & 20.89 & 29.76 & 34.96 & 33.42 & 2.67 & 1.86 \\
\hline 50 & & 29.13 & 37.87 & 33.52 & 32.38 & 2.27 & 1.77 \\
\hline 60 & & 41.54 & 49.51 & 31.97 & 31.21 & 1.93 & 1.61 \\
\hline 70 & & 57.69 & 66.66 & 30.55 & 29.92 & 1.56 & 1.42 \\
\hline 80 & & 77.94 & 83.00 & 29.24 & 28.97 & 1.31 & 1.24 \\
\hline 90 & & 77.94 & 105.45 & 29.24 & 27.93 & 1.31 & 1.11 \\
\hline 100 & & 128.09 & 128.86 & 27.09 & 27.06 & 1.00 & 1.01 \\
\hline
\end{tabular}

\section{Discussion}

As we have discussed earlier, about the proposed architecture, which is shown in Figure 1. Initially the input images are pre-processed using MDBPTGM filter. Here the object image considered and called as "can image" and one more input image as background image that is without can image (object) as shown in Figure 8. This paper aimed at object detection, Hence it is necessary to focus on preserving the object information. Colour images fail to identify and storing the edges of the images, hence it is necessary to convert colour image into grayscale 
images. The input background and object image are converted into grayscale images as shown in Figure 9. Image $\mathrm{C}$ and $\mathrm{D}$ represent the grayscale image of background and object image respectively.

These two input images are pre-processed using MDBPTGM filter and the same are simulated by MATLAB R2012a. Here input images are corrupted with $10 \%$, noisy density of salt and pepper noise. The corrupted background and object images are shown as $\mathrm{E}$ and $\mathrm{F}$ respectively in Figure 10.

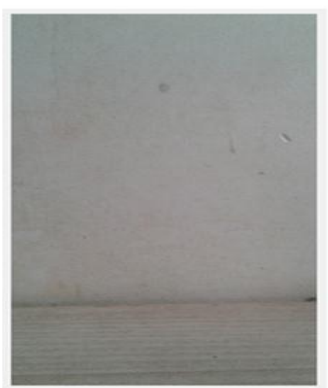

(A)

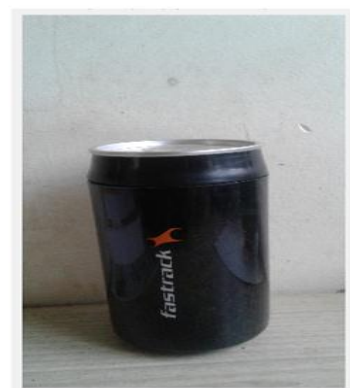

(B)
Figure 8 (A) Original background image (B) Original object (can) image

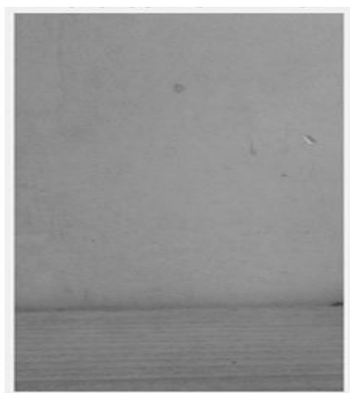

(C)

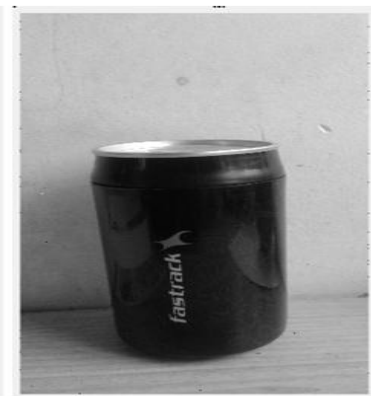

(D)
Figure 9 (C) Background grayscale image (D) Object (can) grayscale image

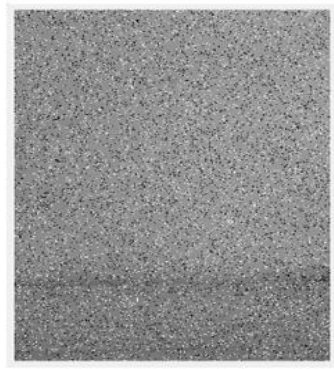

(E)

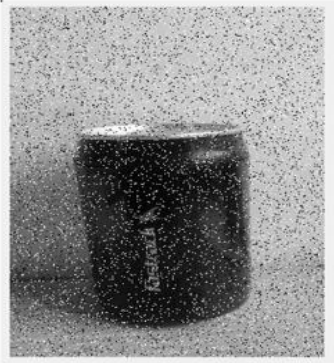

(F)
Figure 10 (E) Background image with salt \& pepper noise (F) Object (can) image with salt \& pepper noise

Input images are filtered by modifying decision based partially trimmed global median filter, out as image 1 and image 2 of filtered background image and filtered object (can image) respectively. The filtered background and object images are shown as $\mathrm{G}$ and $\mathrm{H}$ respectively in Figure 11.

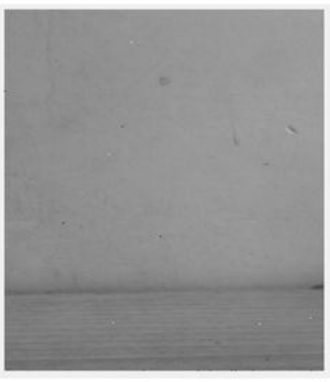

(G)

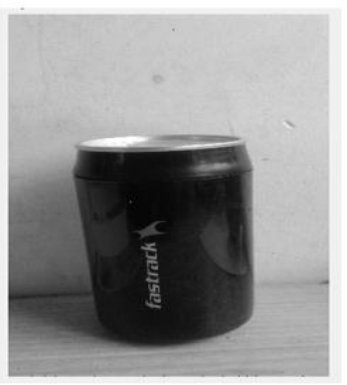

(H)
Figure 11 (G) Filtered background image (H) Filtered object (can) image

Filtered background and object images are applied to the discrete wavelet transform block to extract low frequency sub band (LL band) are shown in Figure 12.

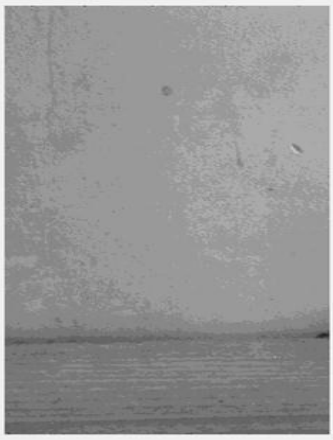

(I)

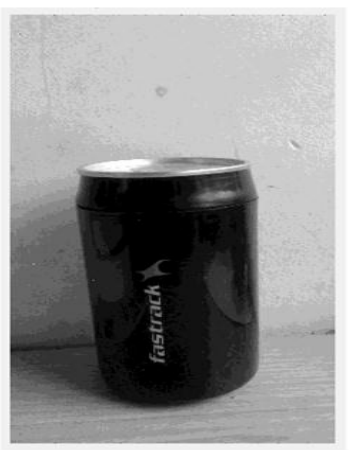

(J)
Figure 12 (I) LL band of background image (J) LL band of object (can) image

The generated LL1 and LL2 bands are used to extract the foreground image, by comparing with adaptive threshold. Adaptive threshold is calculated by Equation 3, the object image is subtracted from the background image pixel by pixel, and the pixel difference between these two is shown in Figure 13 as subtracted image. The subtracted is going to be compared with adaptive threshold values, which is obtained by adding LL band coefficients of object image (LL2) to mean of the subtracted image. Equation 3 gives the adaptive threshold value. It is shown in Figure 14.

If pixel of subtracted image is greater or equal to adaptive threshold value, it will be treated as foreground pixel (black) otherwise it will be remain 
as background pixel (white). The extracted foreground image acts as a detected object image as shown in Figure 15.

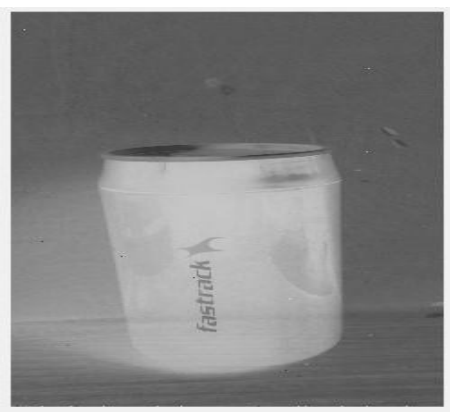

Figure 13 Subtracted image

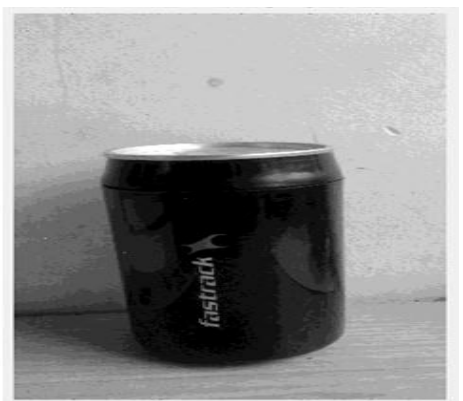

Figure 14 Adaptive threshold image

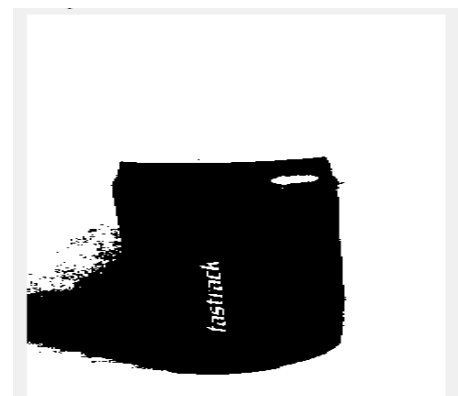

Figure 15 Extracted object image
Similarly, we have conducted the experiment on "Lord Krishna" and "Bottle" images in "JPG" format with $1440 \times 2560$ size which is simulated by MATALB R2012a. Object image initially converted into a grayscale image, then corrupted image filtered by using MDBPTG median filters and finally subtracted from the background. It is defined as the detected object as shown in Table 3. The proposed architecture is a synthesis on Virtex 7 (XC7VX485T) FPGA device by using XILINX ISE design suite 14.5. This summary provides information related to utilization of devices in the proposed architecture. The proposed design uses the 2050 slice registers and 2006 flip-flop pairs, 44 Latches, 1690 slice LUT's and number of shift registers and memory used is 352. The total hardware recourse utilised by proposed architecture is shown in Table 4.

The hardware recourse utilised by proposed architecture is compared with various existing techniques. It is shown in Table 5. The architecture proposed by Qui et al. [10] syntheses on Altera Quartus II 13.1 EDA tool require 9301 slice registers, 3190736 block memory bits and total logic utilised is 5592 out of 32070. Padmanabha et al. [11] Consume 32984 Flip-flops, 29792 LUT's, 77 BRAM, 29792 memory LUT's and 156 DSP48. This architecture synthesised by Xilinx Vivado HLS 2014.4 tool.

The algorithm implemented by Nayak and Pujari [12] on ALTERA Cyclone-II takes 6212 registers, 426 pins, 211872 memory bits. The architecture proposed by SM and Supriya [13] requires 14685 slices, 28818 flip-flops, 28347 LUT's and 15 DSP48 implemented by Xilinx virtex 4 FPGA board.

It is clear that the proposed architecture is efficient in comparison to other methods as shown in Table 5. It uses the least number of hardware parameters, so this design supports reduce power and area usage.

Table 3 Experimental results on Lord Krishna and bottle image

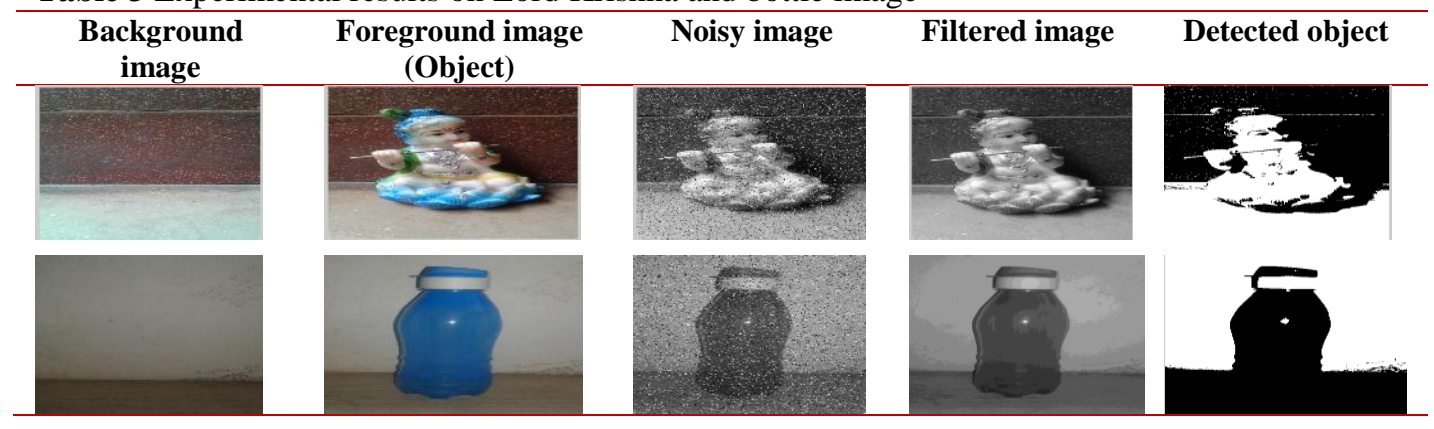


Gangannavar et al.

Table 4 Total resources utilized by proposed architecture

\begin{tabular}{|c|c|c|c|c|c|c|}
\hline $\begin{array}{l}\text { Hardware } \\
\text { resources }\end{array}$ & $\begin{array}{l}\text { Number of slice } \\
\text { registers }\end{array}$ & $\begin{array}{l}\text { Number of flip- } \\
\text { flops pairs }\end{array}$ & $\begin{array}{l}\text { Number of } \\
\text { latches }\end{array}$ & $\begin{array}{l}\text { Number of slice } \\
\text { LUT'S }\end{array}$ & $\begin{array}{l}\text { Number used as } \\
\text { memory }\end{array}$ & $\begin{array}{l}\text { Number used as } \\
\text { shift registers }\end{array}$ \\
\hline Resource used & 2050 & 2006 & 44 & 1690 & 352 & 352 \\
\hline
\end{tabular}

Table 5 Resource utilization comparison

\begin{tabular}{|c|c|c|c|c|c|c|}
\hline $\begin{array}{l}\text { Hardware } \\
\text { Parameters }\end{array}$ & $\begin{array}{l}\text { Qiu et.al } \\
\text { (Cyclone } \\
\text { FPGA) [10] }\end{array}$ & $\mathbf{V}$ & $\begin{array}{l}\text { Padmanabha et al. } \\
\text { (ZYNQ-7000) [11] }\end{array}$ & $\begin{array}{l}\text { Nayak and Pujari } \\
\text { (FPGA- ALTERA } \\
\text { CYCLONE-II) [12] }\end{array}$ & $\begin{array}{l}\text { SM and Supriya } \\
\text { (FPGA-XILINX } \\
\text { VIRTEX4, } \\
\text { XC4FX668-10) } \\
{[13]}\end{array}$ & $\begin{array}{l}\text { Proposed Architecture } \\
\text { (FPGA-XILINX } \\
\text { VIRTEX7 } \\
\text { (XC7VX485T) }\end{array}$ \\
\hline $\begin{array}{l}\text { Number of slice } \\
\text { registers }\end{array}$ & 9301 & & 32984 & 6212 & 14685 & 2050 \\
\hline $\begin{array}{l}\text { Number of flip- } \\
\text { flops pairs }\end{array}$ & - & & - & - & 28818 & 2006 \\
\hline Number of latches & - & & - & - & 28347 & 44 \\
\hline $\begin{array}{l}\text { Number of slice } \\
\text { LUT's }\end{array}$ & - & & 29792 & - & - & 1690 \\
\hline $\begin{array}{l}\text { Number used as } \\
\text { memory }\end{array}$ & - & & - & 211872 & - & 352 \\
\hline $\begin{array}{l}\text { Number used shift } \\
\text { registers }\end{array}$ & - & & - & - & - & 352 \\
\hline
\end{tabular}

\section{Conclusion}

In this paper, a new architecture for object detection based on adaptive threshold has been proposed. It takes the multi-colour and graycolour images corrupted by salt and pepper noise as input, and in simplest segmentation generate the binary image representing the segmentation. The proposed design is a work on modified decision based partially trimmed global median filter. It is well suited for preserving the edges and texture details of an image and performs better for all formats of images. It is observed that the performance parameters like PSNR, MSE and IEF of MDBPTGM filter are better. Hardware parameters such as slice registers, flip-flop pairs, latches, LUT's, shift registers and memory usage are better compared to the other existing techniques. Propose architecture uses the least number of hardware parameters, so the design reduces power, and area usage as compared to other techniques. Future work can focus on overcoming the drawback of increasing or decreasing the window size and the proposed design may be extended for multiple object detection for real time applications.

\section{Acknowledgment}

None.

\section{Conflicts of interest}

The authors have no conflicts of interest to declare.

\section{References}

[1] Del-Blanco CR, Jaureguizar F, García N. An efficient multiple object detection and tracking framework for automatic counting and video surveillance applications. IEEE Transactions on Consumer Electronics. 2012; 58(3):857-62.

[2] Afrose Z. Relaxed median filter: a better noise removal filter for compound images. International Journal on Computer Science and Engineering. 2012; 4(7):1376-82.

[3] Katharotiya A, Patel S, Goyani M. Comparative analysis between DCT \& DWT techniques of image compression. Journal of Information Engineering and Applications. 2011; 1(2):9-17.

[4] Sifuzzaman M, Islam MR, Ali MZ. Application of wavelet transform and its advantages compared to Fourier transform. Journal of Physical Science. 2009; 13: 121-34.

[5] Taneja A, Ranjan P, Ujjlayan A. A performance study of image segmentation techniques. In international conference on reliability, infocom technologies and optimization 2015 (pp. 1-6). IEEE.

[6] Sathua SK, Dash A, Behera A. Removal of salt and pepper noise from gray-scale and color images: an adaptive approach. International Journal of Computer Science Trends and Technology. 2017; 5(1): 117-26.

[7] Sarkar S, Bhairannawar SS, Raja KB, Venugopal KR. An adaptive threshold based FPGA implementation for object and face detection. In international conference on image information processing 2015 (pp. 157-61). IEEE.

[8] Joy NC, Prasad JC. Survey on background modeling and foreground detection methods. International Journal for Innovative Research in Science \& Technology. 2016; 3(4):338-41.

[9] Zaitoun NM, Aqel MJ. Survey on image segmentation techniques. Procedia Computer Science. 2015; 65:797-806.

[10] Qiu D, Sun J, Wu M. Real-time object detection based on the heterogeneous SoC platform. Electrotehnica, Electronica, Automatica. 2017; 65(4):148-54. 
[11] Padmanabha M, Schott C, Robler M, Kriesten D, Heinkel U. ZYNQ flexible platform for object recognition \& tracking. In workshop on positioning, navigation and communications 2016 (pp. 1-6). IEEE.

[12] Nayak S, Pujari SS. Moving object tracking application: FPGA and model based implementation using image processing algorithms. In international conference on computing communication control and automation 2015 (pp. 932-6). IEEE.

[13] SM AR, Supriya MH. Hardware co-simulation of underwater moving object detection using xilinx system generator. International Journal of Oceans and Oceanography. 2016; 10(1):73-80.

[14] Mahajan MP, Jain SN. A survey of non-linear filtering techniques for image noise removal. International Journal on Recent and Innovation Trends in Computing and Communication. 2015; 3(12):68208.

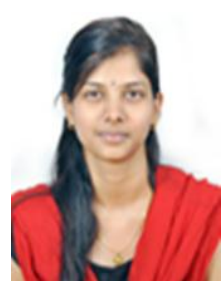

Sangeeta.M.Gangannavar was born in Karnataka, India in 1994. Pursuing M.Tech in Department of Electronics and Communication at SDM college of Engineering and Technology Dharwad, Karnatak. Her main research areas are Image and Video Processing, Digital Electronics, VLSI Design and Embedded System. Email: sangeetaganga01@gmail.com

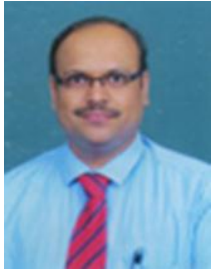

S. S. Navalgund received Bachelor of Engineering in Electronics and Communication Engineering from Karnataka University, Dharwad in 2000 and Master of Technology in Microelectronics and Control Systems from Visvesvaraya Technological University, Belgaum in 2004. Presently he is pursuing the Doctoral studies at VTU, Belagavi. After spending two years in the industry, he joined as a Faculty in N.M.A.M. in Institute of Technology, NITTE, Karnataka. Since 2005, he has been serving as faculty in the Department of Electronics and Communication Engineering, S. D. M. College of Engineering and Technology, Dharwad, Karnataka, India. His main research interests include VLSI Design, Embedded Systems and Digital Signal Processing. He has presented research papers at national/international conferences/journals. He is also a reviewer for many journals. He is currently the member of the Institution of Electronics and Telecommunication Engineer (IETE), Indian Society or Technical Education (ISTE) and Institute of Engineers-India (IE-I).

Email: siddunavalgund@yahoo.com

Satish S Bhairannawar was born in Karnataka, India, in 1979. He received the B.E. degree in Electronics \& Communication from Sir M. Visveshwaraiah Institute of Technology, Bangalore University, Bangalore, India, in 2001. He obtained his M.E in Electronics and Communication Engineering in 2005 from University Visvesvaraya College of Engineering, Bangalore. He was awarded Ph.D for "Efficient VLSI Architectures for Fingerprint Recognition" in 2015, from University Visvesvaraya College of Engineering, Bangalore University.

Email: satishbhairannawar@gmail.com 